\title{
Evaluación de la preferencia para el uso en herramientas gamificadas en la educación superior
}

Evaluation of preference for use of gamificated tools in higher education

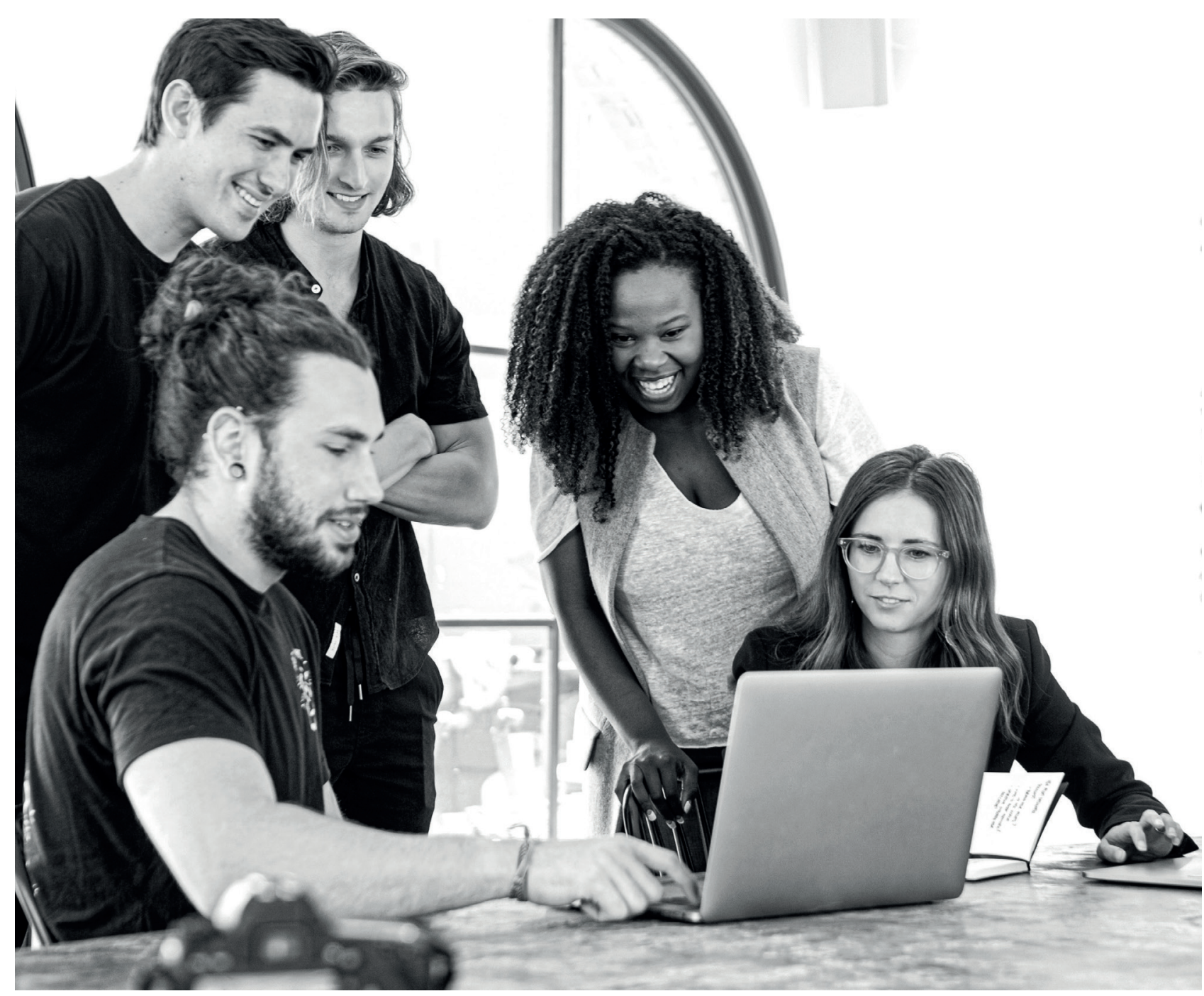

Fuente: Pexels.com Licencia Creative Commons 


\title{
Evaluación de la preferencia para el uso en herramientas gamificadas en la educación superior ${ }^{1}$
}

\author{
Evaluation of preference for use of gamificated tools in higher \\ education
}

\author{
Miguel Ángel Lobo-Rueda², Maira Camila Paba-Medina ${ }^{3}$, Mileidy Alvarez-Melgarejo ${ }^{4}$
}

Artículo recibido en abril 03 de 2020; artículo aceptado en julio 15 de 2020

Este artículo puede compartirse bajo la Licencia Creative Commons Atribución-NoComercial-Compartirlgual 4.0 Internacional y se referencia usando el siguiente formato: Lobo-Rueda, M., Paba-Medina, M. C. y Alvarez-Melgarejo, M. (2021). Evaluación de la preferencia para el uso en herramientas gamificadas en la educación superior. I+D Revista de Investigaciones, 16(1), 17-27. http://dx.doi.org/10.33304/ revinv.v16n1-2021002.

\begin{abstract}
Resumen
Este estudio presenta un recorrido general por el diseño, creación y puesta en marcha de un objeto virtual de aprendizaje (OVA) creado bajo conceptos de la teoría de autogestión y la gamificación para la enseñanza en entornos universitarios, así como su posterior evaluación en términos de preferencia para el uso por parte de los estudiantes. Se parte de la conceptualización de los OVA y las bases teóricas en su construcción, pasando por las metodologías y dinámicas involucradas en su desarrollo, para finalmente mostrar los resultados de las pruebas de preferencia para el uso aplicadas a estudiantes universitarios. Con lo anterior, este OVA y su evaluación se exponen como un caso de estudio único en su tipo al involucrar la gamificación y el uso de este instrumento para abordar la enseñanza en cursos de sistemas de información en un pregrado en ingeniería industrial. Este análisis responde a preguntas como ¿cómo se construye un OVA con conceptos de gamificación? y ¿qué variables influyen en la preferencia del uso de esta herramienta?
\end{abstract}

Palabras clave: Tecnología de la información, método multimedia, sistema de información, gamificación.

\begin{abstract}
This study is a general overview of the design, creation and implementation of a virtual learning object (VLO) created under concepts of self-management theory and gamification for teaching in university environments, as well as its subsequent evaluation in terms of preference for use by students. It starts from the conceptualization of the VLO and the theoretical bases in its construction, then explains the methodologies and dynamics involved in its development, and finally shows the results of the tests of preference for use applied to university students. With the above, this

\footnotetext{
Articulo académico, de enfoque cuantitativo, perteneciente al área de ciencias sociales, subárea de educación, desarrollado en el Laboratorio Galea, grupo de investigación Finance \& Management, financiado por la Universidad Industrial de Santander (Bucaramanga, Colombia). Dirección: carrera 27 calle 9, PBX: (57) (7) 6344000. Fecha de inicio: 12/04/2019. Fecha de terminación: 19/09/2019.

${ }^{2}$ Estudiante de Ingeniería Industrial de la Universidad Industrial de Santander. Vinculado al Laboratorio Galea, Grupo de investigación Finance \& Management, Universidad Industrial de Santander (Bucaramanga, Colombia). Dirección: carrera 27 calle 9, PBX: (57) (7) 6344000. ORCID ID: https:// orcid.org/0000-0002-6618-9024. Correo electrónico: miguelloborueda@hotmail.com.

${ }^{3}$ Estudiante de Ingeniería Industrial de la Universidad Industrial de Santander. Vinculada al Grupo de investigación Finance \& Management, Universidad Industrial de Santander (Bucaramanga, Colombia). Dirección: carrera 27 calle 9, PBX: (57) (7) 6344000. ORCID ID: https://orcid.org/00000002-0453-9367. Correo electrónico: mairacami@hotmail.com.

${ }^{4}$ Estudiante de Maestría en Administración, Universidad de Investigación y Desarrollo. Vinculada al Grupo de investigación Finance \& Management, Universidad Industrial de Santander (Bucaramanga, Colombia). Dirección: carrera 27 calle 9, PBX: (57) (7) 6344000. ORCID ID: https://orcid.org/00000003-1752-8023. Correo electrónico: mileidyalvarezm@gmail.com.
} 
Miguel Ángel Lobo-Rueda, Maira Camila Paba-Medina, Mileidy Alvarez-Melgarejo

Evaluación de la preferencia para el uso en herramientas gamificadas en la educación superior

VLO is presented as a unique case study in its type, involving gamification and the use of this instrument to address the teaching of information systems courses in an undergraduate program in industrial engineering. This analysis answers questions such as: How is an VLO constructed with gamification concepts? And what variables influence the preference for the use of this tool?

Keywords: Information technology, multimedia method, information system, gamification.

\section{Introducción}

La educación como medio de trasformación social y cultural ha cambiado durante el trascurso de la historia humana (Naranjo, 2007). Desde los escribas en Mesopotamia y la tradición oral de los pueblos originarios en América, la educación se ha adaptado a las formas y el contenido del contexto particular de las comunidades. Hoy, en el globalizado y convulsionado mundo contemporáneo, se gesta nuevamente una trasformación en las formas en las que la humanidad desarrolla sus capacidades.

Una realidad cada vez más cercana a la virtualidad ha llevado a la actualización de estrategias y métodos de enseñanza. Lo anterior, debido a que la integración entre las tecnologías de la información y la educación es un hecho, una realidad y una necesidad palpable en los nuevos entornos educativos (Martínez et al., 2018). Prueba de ello es la proliferación en la utilización de entornos virtuales de aprendizaje, como los learning management system (LMS) y la generación de contenidos web educativos (Kakasevski et al. 2008).

Por lo anterior, y a fin de satisfacer la necesidad latente en la incorporación de nuevas tecnologías en las dinámicas educativas (Tedesco, 1998), el Laboratorio de Innovación Educativa GALEA de la Universidad Industrial de Santander creó una experiencia gamificada basada en un objeto virtual de aprendizaje (OVA), bajo las premisas teóricas de la educación experiencial y la teoría de la autodeterminación. Este OVA realiza cambios profundos en la forma en que se abordan temáticas específicas, transformando la distribución y exposición de los contenidos.

Este estudio expone los resultados de la prueba de usabilidad del OVA en un curso de sistemas de información dentro de la carrera de pregrado en ingeniería industrial, y documenta las impresiones de la población objetivo por medio de un instrumento de medición para el registro de 4 variables (motivación, conocimiento, engagement y preferencia para el uso), dentro de un modelo de causalidad. Adicionalmente, exhibe el diseño y el proceso de construcción del instrumento como parte de las necesidades curriculares en la integración de las TIC (Díaz y Torres-Barreto, 2017).

De este modo, se presentan los efectos de una experiencia de gamificación sostenida en un medio como los objetos virtuales de aprendizaje y los resultados de la posible correlación de las variables en el modelo de causalidad subyacente.

\section{Objeto virtual de aprendizaje (OVA)}

Si bien existe una cantidad considerable de definiciones para los objetos virtuales de aprendizaje, numerosos autores coinciden en algunos puntos. Los OVA son entidades digitales soportadas mayoritariamente en computadores y plataformas en línea de carácter versátil, que pueden ser usadas en diferentes contextos (Sánchez, 2014). Son generalmente conformados por recursos virtuales y contenidos con propósito educativo.

Según MinEducación (2009), para Colombia la definición de un objeto virtual de aprendizaje es "un recurso digital que puede ser reutilizado en diferentes contextos educativos. Pueden ser cursos, cuadros, fotografías, películas, videos y documentos que posean claros objetivos educacionales, entre otros" (p. 1). Habitualmente, para la construcción de OVA se crea una amalgama de principios y directrices pedagógicas, combinadas con estándares de calidad. Para lo cual se requiere una estructura básica comprendida por objetivos de aprendizaje, contenido informático, actividades de aprendizaje y una evaluación, tal como se muestra en la Figura 1.

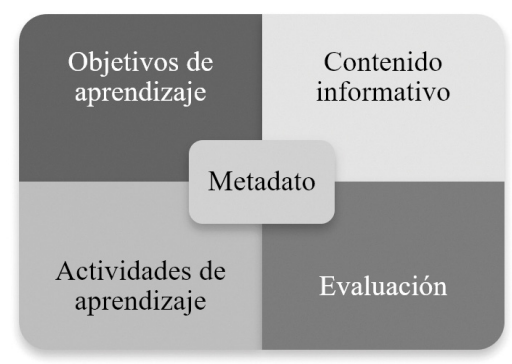

Figura 1. Estructura básica de los Objetos Virtuales de Aprendizaje. Fuente: Indira y Medina (2014).

\section{Base teórica}

El Laboratorio GALEA, gestor del objeto virtual de aprendizaje (OVA) presentado en este estudio, fue fundado bajo las bases de la teoría de la educación experiencial (Lobo-Rueda et al., 2020), la cual sostiene que al controlar el enfoque, la acción, el apoyo, la retroalimentación y la evaluación de la experiencia, se puede garantizar una educación basada en la práctica y el análisis sobre el hacer (Joplin, 1981). Esta teoría, que se ha adaptado a las necesidades particulares del 
Miguel Ángel Lobo-Rueda, Maira Camila Paba-Medina, Mileidy Alvarez-Melgarejo

Evaluación de la preferencia para el uso en herramientas gamificadas en la educación superior

entorno de influencia del laboratorio, contribuyó en el planteamiento y diseño del OVA, al usar las variables previamente mencionadas como base para los estímulos de la experiencia virtual.

De igual forma, la teoría de la autogestión definió la dinámica de uso del OVA, además de la relación del estudiante y el instrumento. Dicha teoría, expuesta por Zimmerman (1989), plantea que el estudiante es el responsable de la diligencia de su propio aprendizaje, participando de manera "metacognitiva, motivacional y conductual" (Góngora, 2002, p. 3) en el proceso.

Lo anterior se traduciría en una participación activa, intensa y persistente del aprendizaje, lo que le otorga un carácter integral, ya que influye en el futuro desempeño laboral. Esta teoría apunta a un cambio de paradigma hacia metodologías de aprendizaje más flexibles, con lo que se aleja de esquemas más rígidos y autoritarios. Esta nueva visión de la interacción en el entorno de aprendizaje genera alumnos más críticos e independientes, de forma que se delega al profesor un rol de guía o monitor, más que de instructor (Rodriguez, 2014).

Finalmente, con el fin de adaptar el OVA a las necesidades de entornos educativos del siglo XXI, se recurrió a la gamificación como estrategia diferencial en la construcción de la herramienta, ya que esta técnica, basada en la adopción de elementos del juego en entornos no lúdicos (Deterding et al., 2011), ha demostrado resultados favorables en la motivación y el engagement en proyectos educativos (Acosta-Medina et al., 2020a).

\section{Modelo de relaciones}

Con el fin de plasmar la experiencia del usuario, se dispuso de un modelo de correlaciones con un enfoque educativo basado en lo realizado por Buitrago-Rodríguez et al. (2018). Para ello, se emplearon las variables de medición usadas por Huizenga et al. (2009), debido a las similitudes en los objetivos del proyecto y el trabajo realizado por estos. Así, se gestó el modelo de causalidad propio de la herramienta, contemplando un indicador (variable observable), la preferencia para el uso, y tres variables (factores): motivación, engagement y conocimiento, como se observa en la Figura 2.

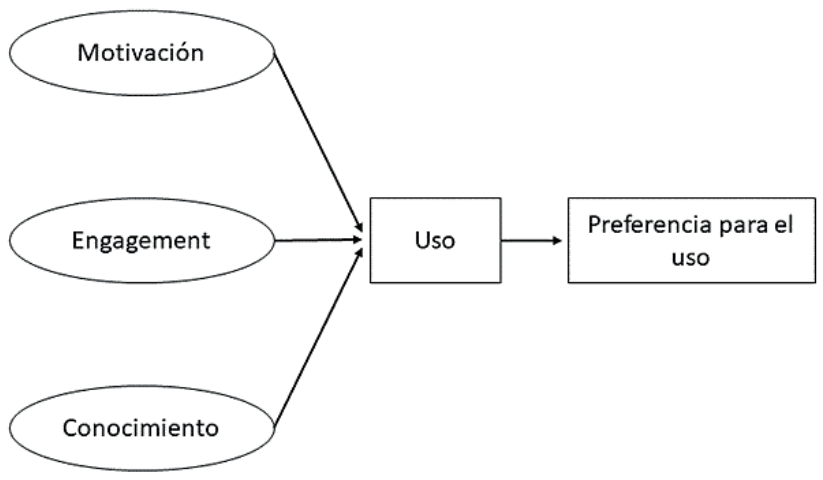

Figura 2. Modelo de causalidad OVA La Orden. Fuente: Autores.

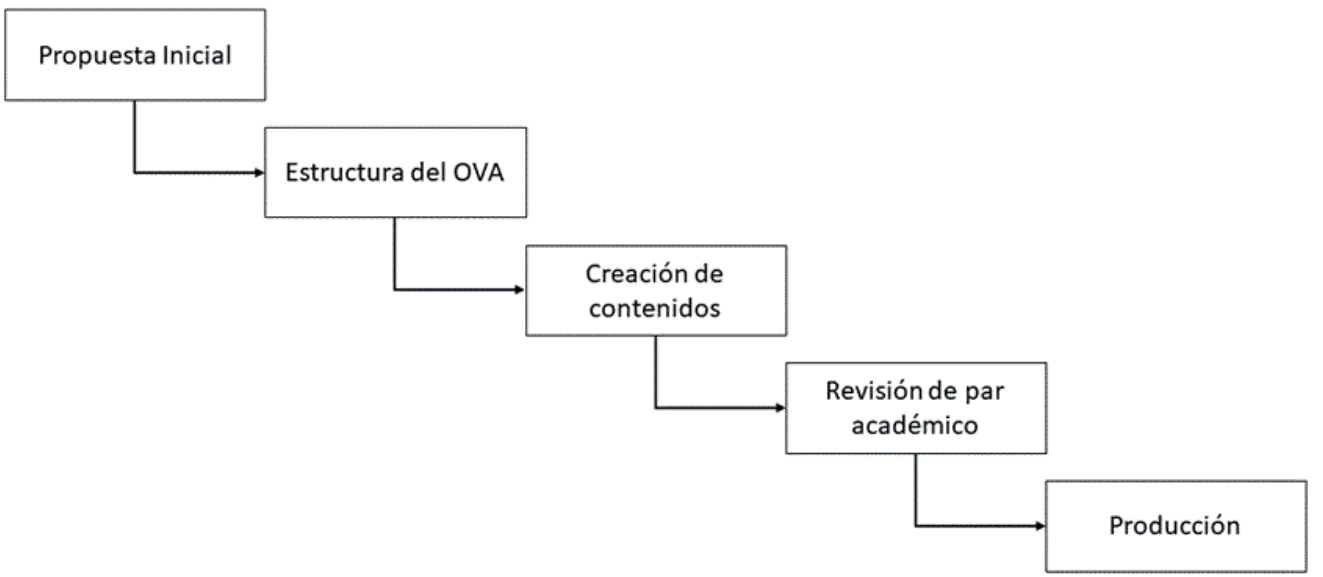

Figura 3. Metodología de construcción de un OVA. Fuente: Autores.

\section{Diseño y producción del OVA}

El método durante el diseño del OVA se basa en el modelo de waterfall para el desarrollo de software (Mahalakshmi y Sundararajan, 2013). Este modelo, al ser adaptado para las necesidades del proyecto, se subdividió en 5 etapas, como se observa en la Figura 3.

En primer lugar, se llevó a cabo la creación del concepto general del OVA, planteando una dinámica de interacción gamificada y una narrativa de storytelling, en busca de una interacción adecuada entre dinámicas, mecánicas y componentes del instrumento (Acosta-Medina et al., 2020b). De este modo se integraron los principios de gamificación planteados por Seaborn y Fels (2015): competencia, autonomía y relacionamiento.

En segundo lugar, fue definida la cantidad de temáticas y el alcance dentro de la asignatura a impactar, lo que dio como resultado la estructura expuesta en la Figura 4, 
Miguel Ángel Lobo-Rueda, Maira Camila Paba-Medina, Mileidy Alvarez-Melgarejo

Evaluación de la preferencia para el uso en herramientas gamificadas en la educación superior

con una limitante en la inclusión de los contenidos de la materia a impactar, con un máximo del $20 \%$.

En tercer lugar, se llevó a cabo la recopilación y creación de los contenidos multimedia, con base en lo definido en la etapa anterior. Posterior a ello, se validaron los contenidos y la estructura pedagógica, por medio de la revisión de un par académico propuesto por el ente financiador del proyecto.

\section{Título OVA Capítulos $\quad$ Subcapítulos}

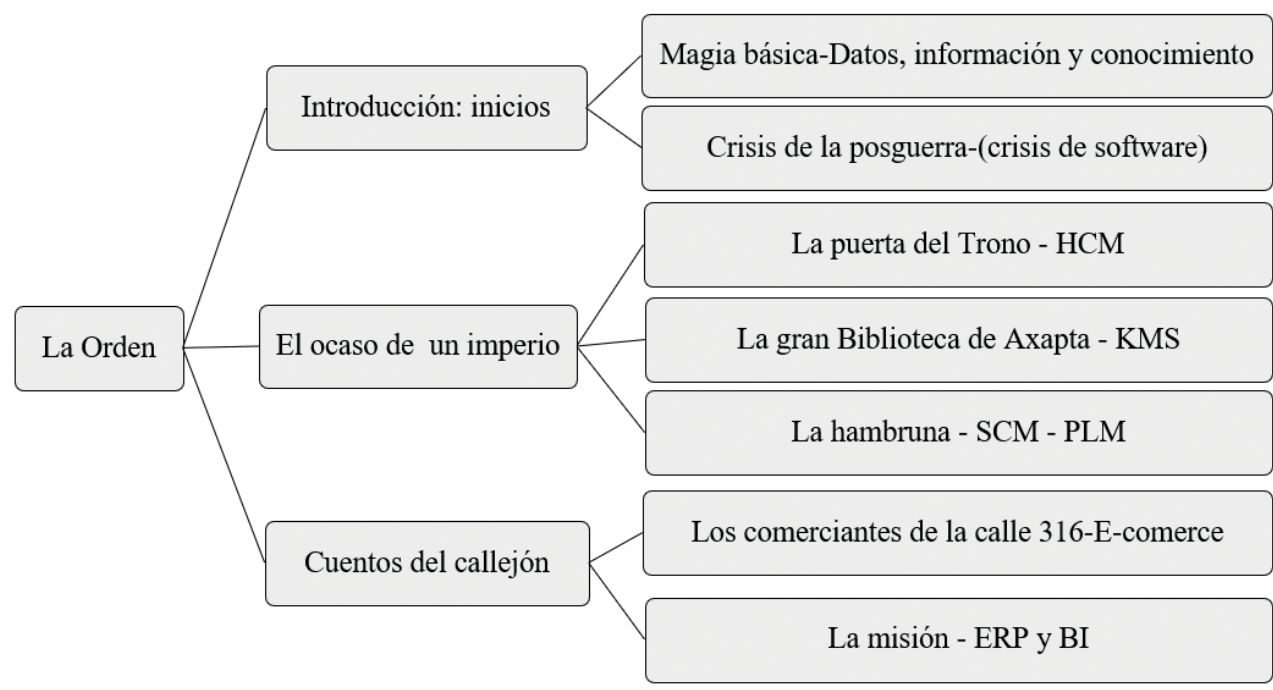

Figura 4. Esquema de un patrón visual complejo. Fuente: Autores.

Como resultado de lo anterior, fue creado el OVA llamado La Orden. Se obtuvo una herramienta virtual portátil con una duración aproximada de 300 minutos de navegación, aplicables a trabajo independiente o complementario dentro del aula, con material que incluye personajes dentro de la narrativa planteada, entornos, videos, locuciones, botones interactivos e imágenes de referencia y vectoriales, como se muestra en la Figura 5.

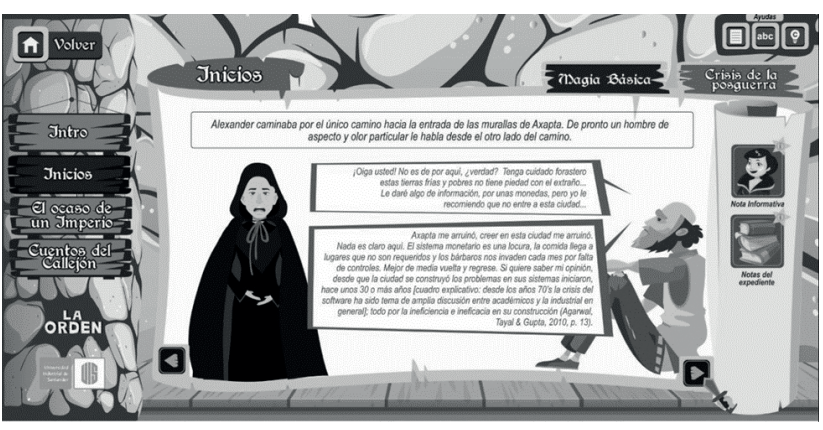

Figura 5. Interfaz del OVA La Orden. Fuente: Autores.

\section{Consideraciones en la construcción del OVA}

El objeto virtual de aprendizaje planteado por GALEA permite la mimetización de contenidos dentro de una narrativa atractiva, lo que facilita una divulgación más
Finalmente, se procedió a la producción del código soportado en Java. Esta última fase se desarrolló por medio de un marco de trabajo SCRUM, gracias a que el componente de gamificación usado en la concepción de la primera fase exigía ajustes y validación de los usuarios. Las pruebas preliminares de la herramienta se realizaron durante la fase final del desarrollo, con un grupo de estudiantes entre los 18 y 23 años.

Magia básica-Datos, información y conocimiento

Crisis de la posguerra-(crisis de software)

La puerta del Trono - HCM

La gran Biblioteca de Axapta - KMS

La hambruna - SCM - PLM

Los comerciantes de la calle 316-E-comerce

La misión - ERP y BI

cercana y cautivadora. Mientras, la enseñanza se abre espacio para un accionar más especializado, gracias al respiro dado por la autogestión de los contenidos por parte del alumnado. Esto implica que el profesorado realice tareas de seguimiento y se enfoque en necesidades educativas no suplidas con métodos tradicionales de aprendizaje. Este acercamiento a una mayor integración de las TIC al desarrollo de contenidos pretende, a largo plazo, modificar la percepción de los actores dentro del proceso de formación. Lo anterior en consonancia con los objetivos y perspectivas actuales de aprendizaje y con los cambios y estrategias necesarias en el siglo XXI (Vélez, 2015).

La dinámica resultante en el OVA La Orden se entiende como el navegar por un diálogo con personajes ilustrados, donde es posible ampliar la información con enlaces o material adicional, dando pequeñas pruebas de conocimiento entre capítulos con el fin de reforzar las habilidades adquiridas y proporcionar un elemento de competencia entre los usuarios. Las ventajas del uso de metáforas y narrativa para el desarrollo de los ejes temáticos de la asignatura posibilitan borrar la barrera existente entre el entretenimiento y la educación formal, lo que aumenta la motivación, concentración y el engagement (Gils, 2005). Por otra parte, al plantear una evaluación orgánica y en sincronía con la narrativa 
Miguel Ángel Lobo-Rueda, Maira Camila Paba-Medina, Mileidy Alvarez-Melgarejo

Evaluación de la preferencia para el uso en herramientas gamificadas en la educación superior

y contenido, bajo contextos no convencionales, se disminuye el nerviosismo propio de metodologías más tradicionales, de forma que es entonces un instrumento innovador para la fluidez del contenido (Falco, 2017).

La construcción de la herramienta enseñó al equipo algunas lecciones para tener en cuenta en proyectos futuros: la comunicación, perspectivas, enfoques en estética y educación diferentes dentro del equipo de trabajo pueden generar retrasos en el desarrollo de la herramienta si se analizan desde la planeación del proyecto. Adicionalmente, se propone revisar la pertinencia del método para el diseño y creación del OVA. Lo anterior debido a que la narrativa dentro del instrumento es atractiva como método no convencional de aprendizaje, pero puede ser tergiversada al usar la metodología propuesta (waterfall). Finalmente, se recomienda controlar la cantidad de texto dentro de un OVA, ya que puede jugar en contra del dinamismo dentro del instrumento, y sin una base de lectura amplia, la información puede perderse al solo presentarse como contenido audiovisual.

\section{Metodología}

Para la realización de este estudio, se tuvieron 3 hipótesis con las que se quiso probar la correlación de las variables y el indicador descrito en el modelo de causalidad. Estas hipótesis se describen a continuación.

H1: Existe una correlación entre la motivación y la preferencia para el uso en el modelo de causalidad.

H2: Existe una correlación entre el conocimiento adquirido y la preferencia para el uso en el modelo de causalidad.

H3: Existe una correlación entre el engagement y la preferencia para el uso en el modelo de causalidad.

Para comprobar estas hipótesis se diseñó un instrumento de medición en formato de encuesta usando una escala de Likert (ver Anexo 1). Para ello, se dividieron las preguntas en 3 categorías para medir las 3 variables y el indicador, distribuidas tal como se muestra en la Tabla 1.

Tabla 1

Distribución de las preguntas asignadas a cada variable o indicador en el instrumento de medición.

\begin{tabular}{ccccc}
\hline Variable/indicador & Motivación & Conocimiento & Engagement & Preferencia para el uso \\
\hline Número de la pregunta & $6,7,15,16,19,20,24$ & $4,12,21$ & $1,2,3,5,11,17,18,22,23,25$ \\
\hline
\end{tabular}

Fuente: Autores. Nota: los números en cada celda hacen referencia a la numeración asignada a la pregunta en el instrumento de medición.

Posterior a ello, se procesaron los datos para probar su correlación, por medio del coeficiente de correlación expresado por la ecuación (Restrepo y Gonzáles, 2007):

$$
\rho_{x y}=\frac{\operatorname{Cov}_{x y}}{\sigma_{x} \sigma_{y}}
$$

Donde:

Cov (x;y): la covarianza entre el valor «x» e «y» $\sigma(x)$ : desviación típica de $x$

$\sigma(y)$ : desviación típica de y

Adicionalmente, se utilizó el coeficiente de correlación de Spearman para, en caso de comprobar alguna hipótesis, verificar si la correlación existente era relevante o, por el contrario, muy débil. Para ello, se utilizó la ecuación (Martínez Ortega et al., 2009):

Donde:

$$
r_{s}=1-\frac{6 \sum d_{i}^{2}}{N\left(N^{2}-1\right)}
$$

di: diferencia entre el i-ésimo par de rangos $=\mathrm{R}(\mathrm{xi})-\mathrm{R}(\mathrm{yi})$ $R$ (xi): rango de $i$-ésimo dato $x$

$\mathrm{R}(\mathrm{yi})$ : rango de i-ésimo dato $\mathrm{y}$
$\mathrm{N}$ : número de parejas de rangos

\section{Público objetivo}

Este estudio se llevó a cabo en un curso de sistemas de información del programa de ingeniería industrial conformado por 35 estudiantes con edades entre los 21 y 34 años, constituido por 21 mujeres (60\%) y 14 hombres (40\%). El grupo donde se aplicó el OVA poseía un promedio académico de 3,8 sobre 5,0 , con una desviación estándar de 0,3.

Este curso se desarrolla bajo un enfoque en el cual se capacita al alumno sobre el uso, construcción, mantenimiento y demás aspectos de los sistemas de información en las empresas. En el momento de realizar la interacción con el objeto virtual los estudiantes contaban con ordenadores separados y conexión a internet para acceder al contenido.

\section{Instrumento de medición}

Para la captura de datos se utilizó un cuestionario de 26 preguntas creadas para medir cada una de las variables por medio de escalas de Likert en una escala de 1 a 5 , donde 1 es totalmente en desacuerdo, y 5, muy de acuerdo. Para la construcción de este cuestionario se tomó lo señalado por Enriquez y Casas (2014) y la 
Miguel Ángel Lobo-Rueda, Maira Camila Paba-Medina, Mileidy Alvarez-Melgarejo

construcción de preguntas propuesta por Almazán y Camus (2013). El cuestionario se puede observar en el Anexo 1.

Tabla 2

Resultados de puntuación por variable e indicador según escala de Likert

\begin{tabular}{cccccc}
\hline Variables & Muy baja & Baja & Media & Alta & Muy alta \\
\hline Motivación & 12 & 38 & 65 & 66 & 54 \\
Conocimiento & 0 & 1 & 19 & 78 & 152 \\
Engagement & 3 & 21 & 76 & 63 \\
Preferencia para el uso & 8 & 42 & 64 \\
\hline
\end{tabular}

Fuente: Autores. Nota: Los datos de la tabla son relativos a cada categoría.
Valoración del engagement en el OVA

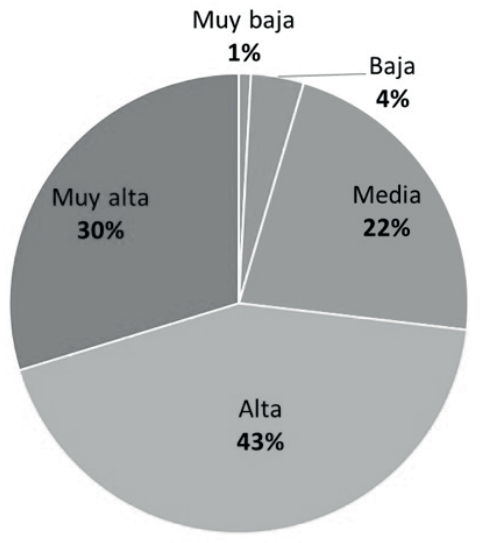

Figura 6. Valoración de la variable engagement en el OVA La Orden. Fuente: Autores.

\section{Valoración de la motivación en el OVA}

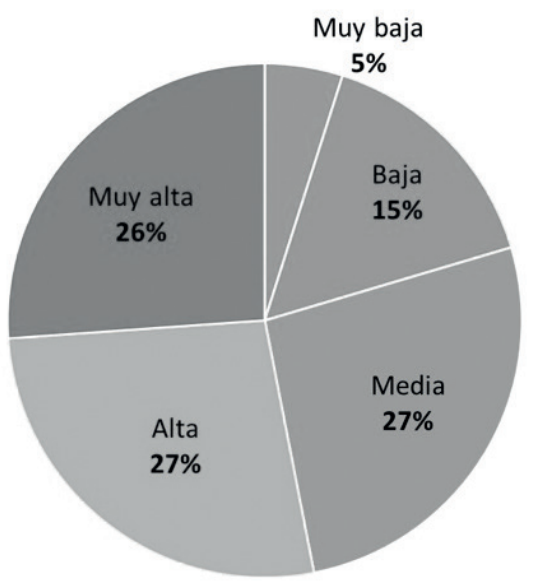

Figura 7. Valoración de la variable motivación en el OVA La Orden. Fuente: Autores.

\section{Resultados y análisis}

Los datos recolectados fueron procesados en el software Microsoft Excel, dando como resultado la Tabla 2, cuyos datos corresponden a cada categoría, por lo que no son comparables de forma directa con los demás. Además, en las Figuras 6,7 y 8 se muestran los resultados por cada variable.

\section{Valoración del conocimiento en el OVA}

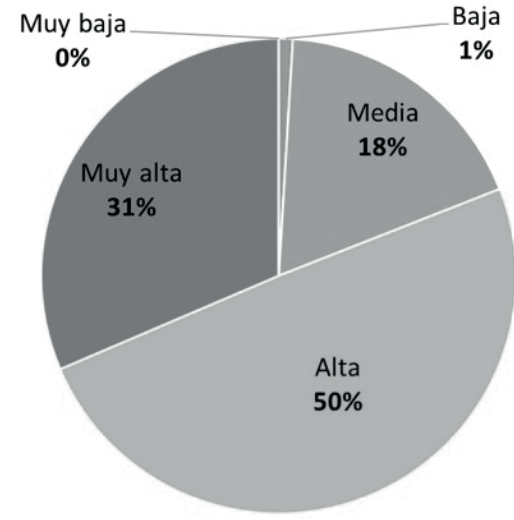

Figura 8. Valoración de la variable conocimiento en el OVA La Orden. Fuente: Autores.

Se evidencia en los tres casos (motivación, conocimiento y engagement) presentados en las Figuras 6, 7 y 8 que predomina la valoración positiva ante la percepción de las variables en el OVA. No obstante, las categorías de valoración alta y muy alta no suman más de un $60 \%$ en la motivación de los estudiantes (ver Figura 7), lo que da cabida a la inacción, ya que poco menos de un tercio de los encuestados dieron una respuesta media. Así, se plantea un cuestionamiento razonable sobre la capacidad de un objeto virtual de aprendizaje para impulsar la acción sobre la autogestión.

En general, según el indicador preferencia para el uso (ver Figura 9), la mitad de los usuarios preferirían la utilización de un objeto virtual de aprendizaje como el OVA La Orden, en lugar de técnicas tradicionales de aprendizaje. No obstante, se observa que más de un tercio de los encuestados no se muestran afectados por la integración de este instrumento en el aula. Por otro lado, las variables de conocimiento y engagement presentaron mediciones similares en el nivel de percepción de los usuarios (ver 
Miguel Ángel Lobo-Rueda, Maira Camila Paba-Medina, Mileidy Alvarez-Melgarejo

Evaluación de la preferencia para el uso en herramientas gamificadas en la educación superior

Figuras 6 y 8), lo que soporta la capacidad del OVA para la transmisión de conocimiento y la generación de fidelidad hacia el instrumento. Como resultado de las mediciones de las variables del modelo, se validan los resultados obtenidos en los índices, ya que, al demostrar que las variables incluidas en las hipótesis se evidenciaron en el OVA, se certifica que los índices son significativos.

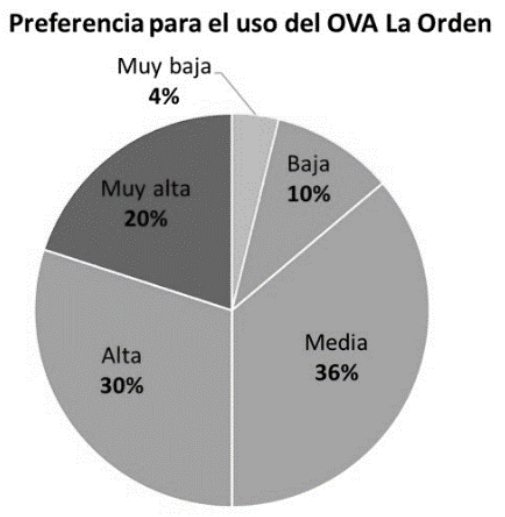

Figura 9. Medición del indicador "Preferencia para el uso" en el OVA La Orden. Fuente: Autores.

Adicionalmente, los resultados mostrados sobre el número requerido de interacciones con el OVA para desarrollar los contenidos arrojaron que los usuarios ingresaron entre 2 y 4 veces a la herramienta (ver Figura 10). Esto indicaría que la mayoría de los estudiantes tardaron entre 2,5 y 1,5 horas en cada interacción, teniendo en cuenta que el instrumento está diseñado para ser explorado en 5 horas (300 min).

\section{¿Cuántas veces requirió entrar al OVA para recorrer su contenido?}

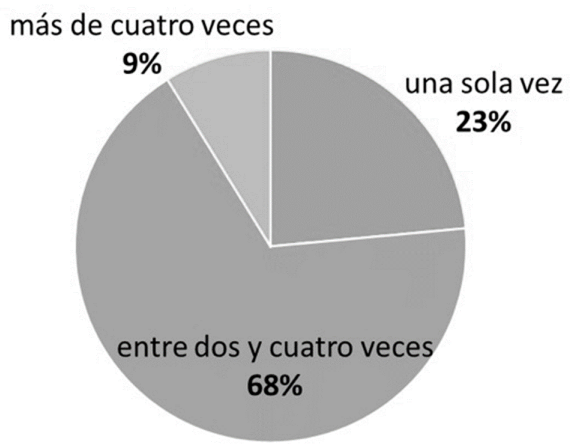

Figura 10. Número de ocasiones en las que el usuario entró al OVA La Orden para completar su desarrollo. Fuente: Autores.

Finalmente, al realizar la comprobación de las hipótesis por medio de los coeficientes de correlación, se encontró que los resultados obtenidos permiten aceptar las hipótesis plateadas $(\mathrm{H} 1, \mathrm{H} 2$ y $\mathrm{H} 3)$, ya que todos los coeficientes muestran un valor diferente de 0 . Así, en la Tabla 3 se observa que todas las correlaciones son de carácter positivo. Sin embargo, al no ser 1 el coeficiente de correlación, solo indica una correlación positiva, sin especificar si es lineal o no. Para complementar el análisis y comprobar si las correlaciones encontradas son matemáticamente relevantes, se utilizó el coeficiente de Spearman. Los resultados de dicho indicador muestran que en todos los casos la correlación es significativa.

Tabla 3

Pruebas de hipótesis

\begin{tabular}{ccc}
\hline Hipótesis & $\begin{array}{c}\text { Coeficiente de } \\
\text { correlación }\end{array}$ & $\begin{array}{c}\text { Coeficiente de } \\
\text { Spearman }\end{array}$ \\
\hline H1 & 0.889562006 & 0.9 \\
H2 & 0.687995399 & 0.7 \\
H3 & 0.772751297 & 0.7 \\
\hline
\end{tabular}

Fuente: Autores.

\section{Conclusiones}

Como resultado del diseño, construcción, validación y monitoreo del OVA La Orden, se encuentra que existe una correlación positiva entre la percepción de motivación, engagement y conocimiento con la preferencia para el uso de los estudiantes al utilizar una herramienta gamificada soportada en plataformas virtuales, como lo es el OVA La Orden.

La construcción y diseño de un objeto virtual de aprendizaje como una herramienta gamificada es adecuada, pues este tipo de OVA puede aportar de manera significativa a la consolidación de temáticas y la modernización de las experiencias de aprendizaje, al mismo tiempo que introduce dinámicas para aumentar el engagement en el proceso formativo. Además, se resalta la importancia de revisar la pertinencia del uso de metodologías tipo waterfall para la construcción de este tipo de plataformas, ya que la naturaleza de la gamificación como estrategia puede dificultar el desarrollo, en términos de tiempo y alcance de este tipo de proyectos.

Finalmente se concluye que la gamificación integrada a la dinámica, mecánica y componentes de instrumentos virtuales de aprendizaje es una acción concreta para la generación de un aprendizaje consciente, lo cual es uno de los retos de la educación soportada en tecnología (Díaz, 2014).

\section{Agradecimientos}

Agradecemos a la Universidad Industrial de Santander, por facilitarnos los escenarios de prueba para la realización de esta investigación, así como a la profesora Martha Liliana Torres Barreto, directora del Grupo de Investigación Finance \& Management y del Laboratorio Galea de esa institución, por su apoyo en el diseño y desarrollo de la herramienta, su supervisión y comentarios para la generación de este manuscrito. 
Miguel Ángel Lobo-Rueda, Maira Camila Paba-Medina, Mileidy Alvarez-Melgarejo Evaluación de la preferencia para el uso en herramientas gamificadas en la educación superior

\section{Referencias}

Acosta-Medina, J. K., Torres-Barreto, M. L. y AlvarezMelgarejo, M. (2020a). Literature Mapping About Gamification in the Teaching and Learning Processes. Revista ESPACIOS, 41(11), 26. https:// www.revistaespacios.com/a20v41n11/20411126. html

Acosta-Medina, J. K., Torres-Barreto, M. L., AlvarezMelgarejo, M. y Paba-Medina, M. C. (2020b). Gamification in the Educational Field : A Bibliometric Analysis. I+D Revista de Investigaciones, 15(1), 30-39.

Almazán, F. y Camus, J. C. (2013). Modelo de test de usuario. Guía Web - Versión 2.0. http://www. guiadigital.gob.cl/guia-v2/capitulos/05/anexos/ pauta-test-usuario.pdf

Buitrago-Rodríguez, J., Tovar-Sánchez, L. y Lamos-Díaz, H. (2018). Modelo de ecuaciones estructurales para el estudio de la percepción de los estudiantes de pregrado de ingeniería industrial con el Proyecto Educativo del Programa-PEP. Revista Educación en Ingeniería, 13(26), 90-100. http://dx.doi. org/10.26507/rei.v13n26.895

Deterding, S., Khaled, R., Nacke, L. E. y Dixon, D. (2011). Gamification: Toward a Definition. CHI 2011 Gamification Workshop Proceedings (Vol. 12). ACM.

Díaz, D. (2014). TIC en educación superior: Ventajas y desventajas. Educación y Tecnología, (4), 44-50. http://revistas.umce.cl/index.php/edytec/article/ view/180

Díaz, A. y Torres-Barreto, M. (2017). Plan para la integración de las TIC en el aula del programa de Ingeniería Industrial de la Universidad Industrial de Santander [trabajo de grado, Universidad Industrial de Santander].

Enriquez, L. y Casas, D. (2014). Usabilidad en aplicaciones móviles. Informes Científicos Técnicos - UNPA, 5(2), 25-47. https://doi.org/10.22305/ict-unpa.v5i2.71

Falco, M. (2017). Reconsiderando las practicas educativas: TICs en el proceso de enseñanza-aprendizaje. Tendencias pedagógicas, 29, 59-79. https://doi. org/10.15366/tp2017.29.002

Gils, F. Van. (2005). Potential Applications of Digital Storytelling in Education. 3rd Twente Student Conference on IT (Vol. 7). University of Twente, Faculty of Electrical Engineering, Mathematics and Computer Science Enschede.

Góngora, J. J. (2002). La autogestión del aprendizaje en ambientes educativos centrados en el alumno. Boletín del Modelo Educativo, Tecnologico de Monterrey.

Huizenga, J., Admiraal, W., Akkerman, S. y Ten Dam, G. (2009). Mobile Game-Based Learning in Secondary Education: Engagement, Motivation and Learning in a Mobile City Game. Journal of Computer Assisted Learning, 25(4), 332-344. https://doi.org/10.1111/ j.1365-2729.2009.00316.x

Sánchez, I. (2014). Estado del arte de las metodologías y modelos de los Objetos Virtuales de Aprendizaje (OVAs) en Colombia. Entornos, (28), 93-107. https:// doi.org/10.25054/01247905.528

Joplin, L. (1981). On Defining Experiential Education. Journal of Experiential Education, 4(1), 17-20. https://doi.org/10.1177/105382598100400104

Kakasevski, G., Mihajlov, M., Arsenovski, S. y Chungurski, S. (2008). Evaluating Usability in Learning Management System Moodle. ITI 2008 - 30th International Conference on Information Technology Interfaces, 36(1), 178-187. https://doi.org/ 10.1109/ ITI.2008.4588480

Lobo-Rueda, M. A., Paba-Medina, M. C. y Torres-Barreto, M. L. (2020). Análisis descriptivo de experiencias gamificadas para enseñanza y aprendizaje en educación superior en ingeniería. Revista ESPACIOS, 41(16), 21. https://www.revistaespacios.com/ a20v41n16/20411621.html

Naranjo, C. (2007). Cambiar la Educación para Cambiar el Mundo (1..$^{\mathrm{a}}$ ed.). Editorial Cuarto Propio.

Mahalakshmi, M. y Sundararajan, M. (2013). Traditional SDLC Vs Scrum Methodology - A Comparative Study. International Journal of Emerging Technology and Advanced Engineering, 3(6), 2-6.

Martínez, R., Gisbert, M. y Larraz, V. (2018). Ecosistemas tecnológicos de aprendizaje y gestión educativa. Edutec. Revista Electrónica de Tecnología Educativa, (64), 1-17. https://doi.org/10.21556/ edutec.2018.64.1025

MinEducación. (2009). Objetos Virtuales de Aprendizaje.

Martínez Ortega, R. M., Tuya Pendás, L. C., Martínez Ortega, M., Pérez Abreu, A. y Cánovas, A. M. (2009). El coeficiente de correlacion de los rangos de Spearman, caracterización. Revista Habanera de Ciencias Medicas, 8(2).

Restrepo, L. y Gonzáles, J. (2007). From Pearson to Spearman. Revista Colombiana de Ciencias Pecuarias, 20(2), 183-192. http:// www.scielo.org.co/scielo.php?script $=$ sci_ 
Miguel Ángel Lobo-Rueda, Maira Camila Paba-Medina, Mileidy Alvarez-Melgarejo

Evaluación de la preferencia para el uso en herramientas gamificadas en la educación superior

arttext\&pid=S0120-06902007000200010

Rodriguez, L. (2014). Cambio de paradigma educativo: del maestro autoritario a la autogestión del alumnado. Iberoamérica Social: Revista-Red de Estudios Sociales, (2), 152-162.

Seaborn, K. y Fels, D. I. (2015). Gamification in Theory and Action: A survey. International Journal of Human-Computer Studies, 74, 14-31. https://doi. org/10.1016/j.ijhcs.2014.09.006

Tedesco, J. C. (1998). Educación y sociedad del conocimiento y de la información. Revista Colombiana de Educación, (36), 36-38.

Vélez, A. (2015). Impacto de las tecnologías de la información en la docencia en educación superior [tesis doctoral, Universidad de León].

Zimmerman, B. J. (1989). A Social Cognitive View of Self-Regulated Academic Learning. Journal of Educational Psychology, 81(3), 329-339. https://doi. org/10.1037/0022-0663.81.3.329 
Miguel Ángel Lobo-Rueda, Maira Camila Paba-Medina, Mileidy Alvarez-Melgarejo

Evaluación de la preferencia para el uso en herramientas gamificadas en la educación superior

Anexo 1: Instrumento de medición para el estudio

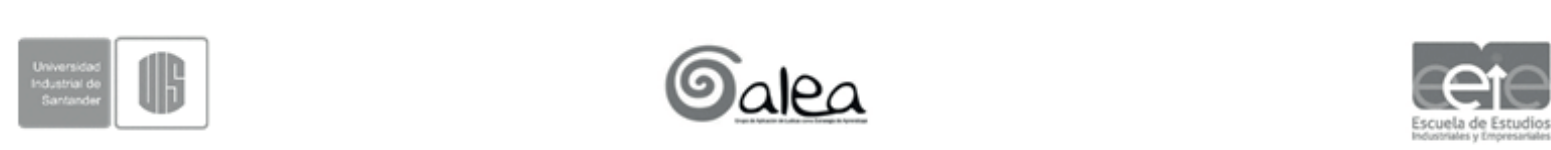

ENCUESTA PARA LA EVALUACION DE FACTORES QUE INCIDEN EN LA PREFERENCIA PARA EL USO DEL OVA LA ORDEN

Estimado estudiante, lasiguiente es unaencuesta con fines de investigación, que busca recolectar información para analizar losfactores que inciden en la preferenciadel uso del Objeto Virtual de Aprendizaje, OVA - La Orden. Agradecemos su voluntady

EDAD:

CARRERA:

GÉNERO:

$(\mathrm{M} / \mathrm{F})$

Por favor marque con una EQUIS $(X)$ el número de veces que ingresó al OVA, con el fin de recorrer sus contenidos

1. Nunca ___ 2. En una sola vez hice todo el recorrido del OVA _ _ Necesité entre dos y cuatro veces ___

4. Necesité más de 4 veces __ $\quad 5$. Necesité más de siete veces en total___

Estimado estudiante, por favor responda lasiguiente encuestamarcando con una " $\mathrm{X}$ " el número según su pr efe encia de la siguiente mane a: $\begin{array}{llll}\text { 1-Totalmente en desacuerdo } 2 \text {-En desacuer do } \quad 3-\mathrm{Ni} \text { en acuerdo ni en desacuer do } & \text { 4. Deacuerdo } & \text { 5.Muyde acue do }\end{array}$ 